\title{
Defect detection in periodic patterns using a multi-band-pass filter
}

\author{
Zong-Da Tsai · Ming-Hwei Perng
}

Received: 30 May 2011 / Revised: 11 January 2012 / Accepted: 13 March 2012 / Published online: 11 May 2012

(C) The Author(s) 2012. This article is published with open access at Springerlink.com

\begin{abstract}
This study presents a rapid and reliable technique for the inspection of defects in a two-dimensional periodic image using a multi-band-pass filter. More importantly, the blurring effect of the resultant defect images is significantly reduced, thereby resulting in a more precise estimation of the size of defects when compared with methods that use low-pass filtering. As a filter-based approach, the present technique does not require an alignment procedure. In addition, computational time is reduced by implementing multiband-pass filters with convolution masks when the filters are operated in the spatial domain. Further, this approach involves mostly addition operations with very few multiplications; hence, computational time is significantly reduced when compared with those for existing approaches. The efficiency and effectiveness of the proposed multi-band-pass filter is verified through examples. It is observed that there is a significant reduction in blurring effects, leakage effects, and computational effort. It is noteworthy that though the proposed approach is presented as a two-dimensional filtering problem, it can be reduced to a one-dimensional filtering problem under the assumption that the misorientation angle of the inspected periodic pattern is negligibly small.
\end{abstract}

Keywords Automatic optical inspection - Computer vision - Defect detection - Multi-band-pass filter .

Periodic pattern

\section{Z.-D. Tsai $(\varangle) \cdot$ M.-H. Perng}

The Department of Power Mechanical Engineering,

National Tsing Hua University, 101 Sec. 2,

Kuang-Fu Road, 30013 Hsinchu, Taiwan, ROC

e-mail: zdtsai@msn.com

\section{Z.-D. Tsai}

The National Synchrotron Radiation Research Center, 101, Hsin-Ann Rd., Science-Base Industrial Park,

30076 Hsinchu, Taiwan, ROC

\section{Introduction}

Due to rapid growth in the batch production processes in the semiconductor industry, automatic optical surface inspection techniques used to detect defects (e.g., defects in semiconductor wafers and integrated circuits) have become increasingly important. These inspection techniques require high image resolution, high precision, and high computational speeds. Automated inspection systems scan the devices under inspection as periodic patterns. Defect inspection techniques in two-dimensional (2D) periodic patterns have a wide range of industrial applications in areas such as memory chips, shift registers, switched capacitors, charge-coupled device (CCD) arrays, and liquid crystal displays (LCDs).

Most existing approaches for defect inspection in periodic patterns can be divided into three main classes: (1) template matching [1-13]; (2) neural networks [14-20]; and (3) filter approaches [21-33]. A few other studies have reported approaches other than the abovementioned [34].

The template-matching process is divided into two categories. The traditional template matching method uses an external golden template created by the image registration and segmentation process to discriminate defects [2-4]. An alternative approach is the die-to-die inspection method [4-12] that can be considered as a special form of the template-based approaches that have been adopted in many industrial applications. This approach is well-known for its simplicity and computational efficiency. In this approach, identical areas on two or more neighboring dies are compared based on the differences that are caused by all the defects and noises present in the dies. However, both these approaches require precise alignment and segmentation of the periodic patterns to find the corresponding pixels in each die. Further, the required precisions increase with the density of the periodic patterns [10-13]. Moreover, as the sizes of the defects decrease 
(making defect detection much more difficult), these processes can become fairly complicated and computationally demanding.

In approaches using neural networks and statistical methods [14-20], it is difficult to discriminate the defective area with periodic backgrounds. Therefore, neural networks have limited applications in the mura inspection of thin film transistor-liquid crystal displays (TFT-LCDs) $[14,15]$ and in the relatively simple patterns of light emitting diodes (LEDs) $[16,17]$. In particular, the support vector data description (SVDD) method using a fuzzy penalty function has been proposed to inspect defects in gate electrodes $[15,18]$. However, this method is valid only for inspecting defects with a clear background. Two previous studies have reported remarkable increases in defect discrimination ability by repeatedly training cellular neural networks [19,20]. However, it is timeconsuming to collect a sufficient number of typical trained patterns (as required in the above approaches) with normal and abnormal images in actual applications. Besides, small defects escape detection easily because the cellular neural network cannot learn to describe an accurate shape. Therefore, these methods cannot meet current requirements in industrial applications.

The third class of detection is the use of filter-based techniques [21-33] such as the Gabor filter [21-24], the wavelet transform [25-27], and the discrete Fourier transform (DFT) [28-33]. The Gabor filter uses a Gaussian-type bandpass filter to extract defects; here, the defect spectrum must be exactly mapped in order to extract the defects [21-24]. The wavelet transform approach uses different order bandpass filters and down-sampling techniques to separate the background of periodic patterns. In addition, the approach may be used in the pre-processing stage of defect inspection [25-27]. However, the processes of forward and backward wavelet transforms usually consume more time than the permitted inspection time in the semiconductor industry. The Discrete Fourier Transform (DFT) is also used to separate the background of periodic patterns; this involves less computational effort than the aforementioned filter-based techniques [28-33]. The DFT has been applied to discriminate a defective textile from a good one by analyzing its frequency spectrum [24, 28, 32]. However, none of these reported studies has ever attempted to determine the location and shape of defect areas in periodic patterns although this is a common requirement in the automatic optical inspection (AOI) and the automatic vision inspection (AVI) industries.

Some previous authors have attempted to locate defect areas and their shapes in a periodic pattern using a 1D or a 2D low-pass filter [29-31]. In these approaches, the DFT is applied first to an image containing the periodic patterns; a $1 \mathrm{D}$ or a $2 \mathrm{D}$ low-pass filter is subsequently designed to eliminate the periodic patterns, resulting in the extraction of the defect areas. This approach assumes inherently that the periodic patterns are confined to the medium- and high-frequency spectrum; the defect spectrum is thereby assumed to be limited to the low frequency range, so introducing blurring effects on the defects.

In the light of this assumption, the size of the defect areas must be sufficiently large, and the periodic patterns must have very strong contrast and a relatively small period. Otherwise, the spectrum of the defect areas may seriously overlap with the periodic patterns, causing difficulties in choosing an appropriate threshold. Besides, all the transformations techniques between the spatial domain and the frequency domain are computationally demanding and time consuming; therefore, they fail to meet the requirements of high speed and high resolution inspections required in certain industrial applications.

In order to meet the demands of increasingly high resolution and high-speed inspection techniques, we have developed an approach that uses multi-band-pass filters with a pattern padding technique and a $2 \mathrm{D}$ convolution mask for the inspection of defects in periodic patterns; this approach is based on a preliminary study carried out by one of us [35]. Our approach in this study mainly consists of two steps. First, a pattern padding technique is introduced to reduce the leakage effect during DFT process. Second, a multi-band-pass filter is designed to sharply separate the periodic pattern from the defect patterns. This approach has the following advantages: (1) the total computational effort is significantly less than that of most frequency domain approaches due to the usage of convolution masks, and it is of the same order as that of the basic die-to-die inspection method; (2) there is no need to perform alignment, segmentation, and golden template generation; (3) the filter can detect defect areas more sharply than most of the existing techniques; and (4) the present method robustly resists errors caused by changes in the luminosity and the orientation of the test image.

This paper is organized as described below. First, the characteristics and the frequency spectrum of a $2 \mathrm{D}$ periodic pattern are thoroughly investigated in Sect. 2. This is done to show the necessity of inspecting the periodic pattern in two dimensions rather than reducing it into a one-dimensional (1D) problem in the presence of angular misorientation. In addition, the effectiveness of using a multi-band-pass filter over a low-pass filter is shown. Subsequently, Sects. 3.1 and 3.2 show that multi-band-pass filtering and the pattern padding technique can be used to significantly reduce blurring effects caused by a traditional low-pass filter. Section 3.3 presents the design of a convolution mask to approximate the multi-band-pass filter; the mask is directly applied to the test image with the periodic pattern image. Further, it is shown that the proposed convolution mask can realize the multi-band-pass filter in the spatial domain and significantly reduce computational effort. Sect. 3.4 describes the numerical efficiency of the present approach. Finally, Sect. 4 
gives examples to verify the performance of the proposed approach, and Sect. 5 concludes this paper.

\section{Effects of misorientation and leakage}

This section discusses two main effects-misorientation of the inspected device and energy leakage during the DFT process - with respect to defect detection within periodic patterns. Section 2.1 shows that in the presence of device misorientation, a 2D periodic pattern should not be treated as a 1D periodic signal. Further, the leakage phenomenon owing to the windowing effects of a discrete signal will be thoroughly investigated in Sect. 2.2 to reveal the shortcomings of prevailing low-pass filtering techniques in defect detection.

\subsection{Effects of misorientation}

Some researchers have used 1D filtering techniques to detect defects in a 2D pattern to simplify the task of detection $[29,30]$. However, in this section we shall show that the analysis of a $2 \mathrm{D}$ image should not be replaced by that of a 1D image in the presence of angular misorientation.

Let $p(\bar{x}, \bar{y})$ be a signal defined in the interval $\bar{x} \in$ $\left[0, T_{x}\right], \bar{y} \in\left[0, T_{y}\right]$, where the spatial periods are $T_{x}$ and $T_{y}$, and $p(\bar{x}, \bar{y})$ repeats itself in $f(x, y)$. That is,

$f(x, y)=p(\bar{x}, \bar{y})$

if and only if $x=\bar{x}+m T_{x}$ and $y=\bar{y}+n T_{y}$. Here, $\mathrm{m}$ and $\mathrm{n}$ are integer values $(m=0,1,2, \ldots$ and $n=0,1,2, \ldots)$. Let us assume that $f(x, y)$ represents a 2D periodic signal with a number of samples. Because of misorientation of the inspected object, the periodic patterns now appear to have new periods $T_{x}^{\prime}$ along the $x^{\prime}$ axis and $T_{y}^{\prime}$ along the $y^{\prime}$ axis. As shown in Fig. 1, the new period $T_{x}^{\prime}$ is the length of the horizontal dashed line between the two points in the repeated

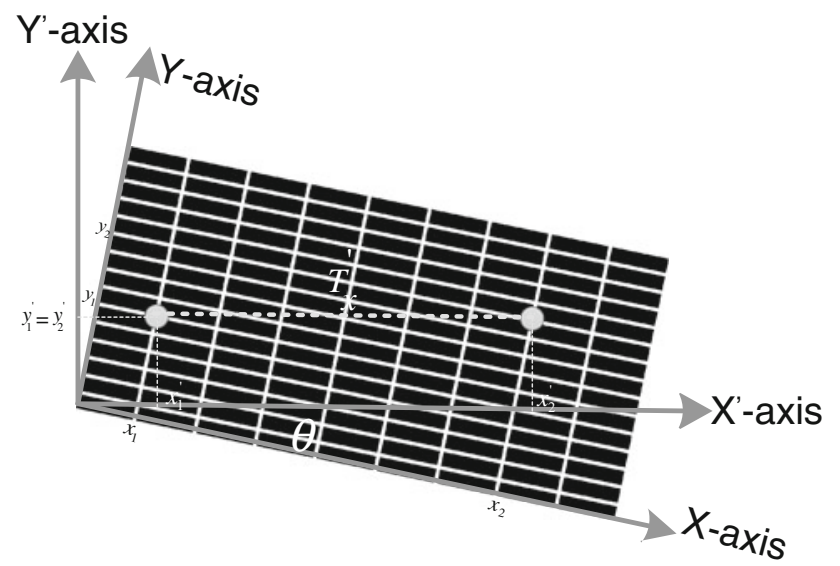

Fig. 1 Periodic patterns with tilt angle signal location. The dashed line is at an angle $\theta$ with respect to the $x$ axis in the counterclockwise direction. The two points $\left(x_{1}, y_{1}\right)$ and $\left(x_{2}, y_{2}\right)$ have now shifted to the new coordinates $\left(x_{1}^{\prime}, y_{1}^{\prime}\right)$ and $\left(x_{2}^{\prime}, y_{2}^{\prime}\right)$, respectively, because of the rotation of the $2 \mathrm{D}$ Cartesian coordinates. The relation between the rotation angle $\theta$ and the periods is shown in (2a) and (2b) as below

$$
\begin{aligned}
& \left\{\left|\begin{array}{l}
x_{1}^{\prime} \\
y_{1}^{\prime}
\end{array}\right|=\left|\begin{array}{cc}
\cos \theta & \sin \theta \\
-\sin \theta & \cos \theta
\end{array}\right|\left|\begin{array}{l}
x_{1} \\
y_{1}
\end{array}\right|\right. \\
& \left\{\left|\begin{array}{l}
x_{2}^{\prime} \\
y_{2}^{\prime}
\end{array}\right|=\left|\begin{array}{cc}
\cos \theta & \sin \theta \\
-\sin \theta & \cos \theta
\end{array}\right|\left|\begin{array}{l}
x_{2} \\
y_{2}
\end{array}\right| .\right.
\end{aligned}
$$

Here, the coordinates $\left(x_{1}, y_{1}\right)$ and $\left(x_{2}, y_{2}\right)$ are given by $x_{1}=$ $m_{1} T_{x}+\bar{x}, y_{1}=n_{1} T_{y}+\bar{y}$ and $x_{2}=m_{2} T_{x}+\bar{x}, y_{2}=$ $n_{2} T_{y}+\bar{y}$, respectively, where $m_{1}, m_{2}, n_{1}$ and $n_{2}$ are integer values. The two points located on the horizontal dashed line related to the new coordinates have the same value along the $Y^{\prime}$ axis. Because $y_{2}^{\prime}=y_{1}^{\prime}$ in the repeated signal location and $\bar{m}=m_{2}-m_{1}, \bar{n}=n_{2}-n_{1}$, we obtain the relation

$\bar{n} T_{y}=\bar{m} T_{x} \tan \theta$.

It is clear that when $\theta$ is specified such that $\tan \theta \approx 0.5$, (3) reduces to

$\bar{n} T_{y}=0.5 \bar{m} T_{x}+\delta$,

and $\delta$ denotes the approximation in (4) within an error bound. It is possible to find $\bar{m}, \bar{n}$ such that they are almost integer values for a specific value of $\theta$. Further, if $\theta$ is not negligibly small, the new periods $T_{x}^{\prime}$ are generally different from the original period $T_{x}$. However, it is difficult to find integer values for $\bar{m}, \bar{n}$ in the bounded image size. In such cases, the result of defect detection using a $1 \mathrm{D}$ filtering technique will fail.

\subsection{Effects of leakage}

In most previous studies, such as [29-31], it has been assumed that the inherent energy in a periodic pattern is concentrated in the high frequency range, while all the energy present in the lower frequency range are assumed to be due to defects. However, the actual energy distribution of a periodic pattern is more complicated than the aforementioned assumption due to reasons such as leakage effects or the Gibbs phenomenon [37]. This subsection is devoted to the study of the energy distribution pattern of a periodic image in order to derive a better approach for defect detection.

Let $\widehat{f}(n)$ be a $1 \mathrm{D}$ discrete signal. Its finite length signal $f(n)$ with a finite window length $L$ be expressed as

$$
f(n)=\widehat{f}(n) w(n)
$$


The window function $w(n)$ is given as

$w(n)=\left\{\begin{array}{ll}1, & 0 \leq x \leq L-1 \\ 0, & \text { otherwise }\end{array}\right.$.

The Fourier transform of this function is

$W(\omega)=\frac{\sin (\omega L / 2)}{\sin (\omega / 2)} \mathrm{e}^{-j \omega(L-1) / 2}$.

Therefore, if we have a periodic image with the image size $N_{x} \times N_{y}$, the DFT of the discrete periodic signal $f(x, y)$ will be given by

$f(x, y)=\widehat{f}(x, y) w(x, y)$.

The windowing function $w(x, y)$ is a 2D generalization of the classical windowing function,

$w(x, y)= \begin{cases}1, & 0 \leq x \leq N_{x}-1 \text { and } 0 \leq y \leq N_{y}-1 \\ 0, & \text { otherwise }\end{cases}$

whose Fourier transform is

$$
\begin{aligned}
W(u, v)= & \frac{\sin \left(u N_{x} / 2\right)}{\sin (u / 2)} \mathrm{e}^{-j u\left(N_{x}-1\right) / 2} \\
& \cdot \frac{\sin \left(v N_{y} / 2\right)}{\sin (v / 2)} \mathrm{e}^{-j v\left(N_{y}-1\right) / 2},
\end{aligned}
$$

where $u=2 \pi / T_{x}$ and $v=2 \pi / T_{y}$ and $W(u, v)$ is responsible for the leakage effect wherever $W(u, v)$ is valid. Substituting $u=2 \pi / T_{x}$ and $v=2 \pi / T_{y}$ into (10) and eliminating $u$ and $v$, we obtain

$$
\begin{aligned}
W(u, v)= & \frac{\sin \left(\pi N_{x} / T_{x}\right)}{\sin \left(\pi / T_{x}\right)} \mathrm{e}^{-j \pi\left(N_{x}-1\right) / T_{x}} \\
& \cdot \frac{\sin \left(\pi N_{y} / T_{y}\right)}{\sin \left(\pi / T_{y}\right)} \mathrm{e}^{-j \pi\left(N_{y}-1\right) / T_{y}} .
\end{aligned}
$$

Equation (11) indicates that $|W(u, v)|=0$ and the leakage effect vanishes whenever either $N_{x} / T_{x}=n_{x}$ or $N_{y} / T_{y}=$ $n_{y}$, where $n_{x}$ and $n_{y}$ are integer values. Besides, $|W(u, v)|$ and the leakage effect reach their maxima at $N_{x} / T_{x}=\left(2 n_{x}+\right.$ 1) $/ 2$ or $N_{y} / T_{y}=\left(2 n_{y}+1\right) / 2$ for given integer values of $n_{x}$ and $n_{y}$.

For instance, consider an image $f(x, y)$ with periodic patterns shown in Fig. 2a, where $f(x, y)$ consists of $N_{x} \times N_{y}=$ $256 \times 256$ pixels while the periods are $T_{x}=8$ pixels and $T_{y}=16$ pixels. The gain plot of the DFT of $f(x, y)$ is shown in Fig. 2b, where the leakage effect does not occur owing to the fact that $|W(u, v)|=0$ for all $u$ and $v$ when $N_{x} / T_{x}=32$ and $N_{y} / T_{y}=16$. Let us now assume that $f(x, y)$ remains the same but the period $T_{y}=16$ is replaced by $T_{y}=17$. Consequently, leakage occurs along the horizontal direction in the gain plot of the DFT of $f(x, y)$ as shown in Fig. $2 \mathrm{c}$; this is due to the fact that $N_{y} / T_{y}$ is no longer an integer value. Further, if $T_{x}=7$ pixels and $T_{y}=17$ pixels, leakage occurs along both the horizontal and vertical directions in the gain plot of the DFT of $f(x, y)$, as shown in Fig. 2d. Neither $N_{x} / T_{x}$ nor $N_{y} / T_{y}$ is an integer values.

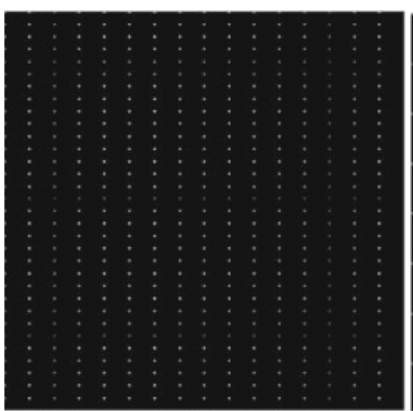

(a)

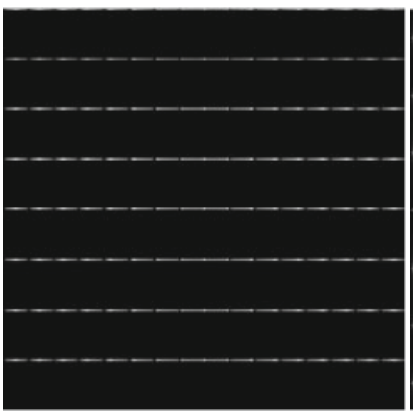

(c)

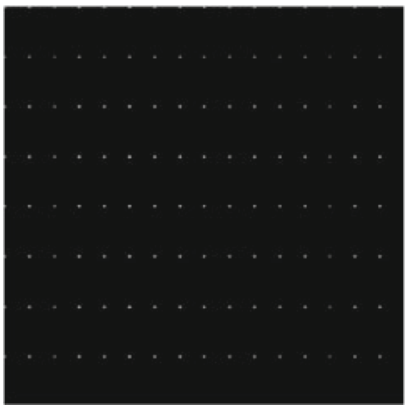

(b)

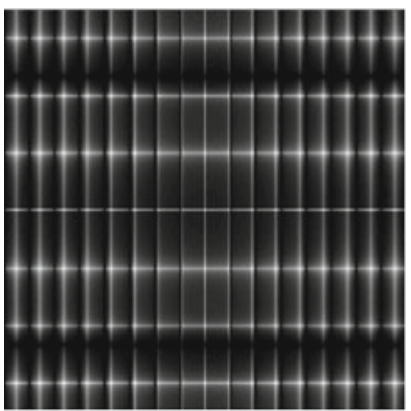

(d)
Fig. 2 Discrete Fourier transform (DFT) of $f(x, y)$ with leakage effects. a Original image $f(x, y), \quad N_{x} \times N_{y}=256 \times 256$. b DFT of $f(x, y), T_{x}=8, T_{y}=16$. $\mathbf{c}$ DFT of $f(x, y), T_{x}=8, T_{y}=17$. d DFT of $f(x, y), T_{x}=7, T_{y}=17$

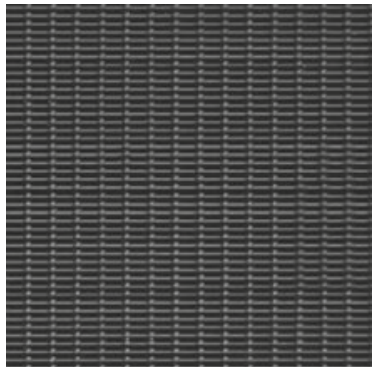

(a)

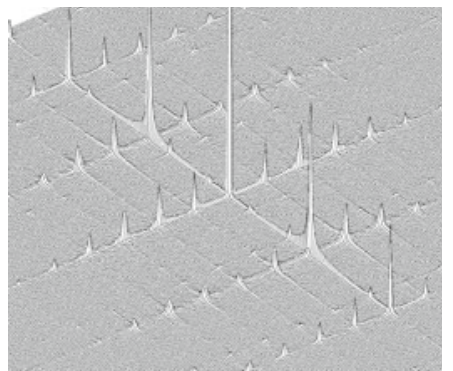

(b)
Fig. 3 Leakage spectrum of liquid crystal display (LCD) array. a Image of a LCD array $f(x, y)$. b Spectrum of the LCD array $|F(u, v)|=|\Im\{f(x, y)\}|$

In many applications of signal processing, this leakage is treated as unwanted noise to be filtered out. In the case of AOI, leakage is an essential part of a 'normal image'; it should not be filtered out in the generation of a golden template. For instance, Fig. 3 shows the real image of a TFT-LCD panel and its spectrum with leakage effects. The image size in Fig. $3 \mathrm{a}$ is $N_{x} \times N_{y}=256 \times 256$ pixels while the periods of the pattern are $T_{x}=6$ pixels and $T_{y}=17$ pixels. The height axis in Fig. $3 b$ indicates the spectrum energy of the image in Fig. 3a at coordinates $(u, v)$ in the frequency domain. The highest peak occurs at the location $(u, v)=(0,0)$ and the other peaks correspond to periods that are integer multiples of the fundamental frequency at $(u, v)=\left(n_{x} \omega_{x}, n_{y} \omega_{y}\right)$, 
with $n_{x}$ and $n_{y}$ taking integer values. The leakage effect must be handled carefully to obtain a sharp image of the defects in the pattern.

\section{Defect detection using pattern padding and multi-band-pass filter technique}

This section proposes an multi-band-pass filter to accurately extract defects from a periodic pattern. Section 3.1 discusses the use of a multi-band-pass filter for defect detection in a periodic pattern. In Section 3.2, the performance of this multiband-pass filter is shown to be enhanced using a padding technique to minimize the leakage energy. In order to minimize computational effort, the proposed multi-band-pass filter is realized as a spatial convolution mask; this is described in Sect. 3.3. Section 3.4 compares the numerical efficiency of the present approach against those using DFT and Fast Fourier Transform (FFT).

\subsection{Defect detection by multi-band-pass filtering}

In this section, a new approach using a 2D DFT is presented for the reconstruction of a golden template from a possibly defective periodic pattern. The requirement is to sensitively and sharply discriminate the periodic pattern from defects to obtain the precise location and shape of the defect areas.

Let $f(x, y)$ be a 2D periodic signal with a finite number of samples. The DFT of a function $f(x, y)$ of image size $N_{x} \times N_{y}$ is given by the equation

$$
\begin{aligned}
F(u, v) & =\Im\{f(x, y)\} \\
& =\sum_{x=0}^{N_{x}-1} \mathrm{e}^{-j 2 \pi u x / N_{x}} \sum_{y=0}^{N_{y}-1} f(x, y) \mathrm{e}^{-j 2 \pi v y / N_{y}}
\end{aligned}
$$

for $u=0,1,2, \ldots N_{x}-1$ and $v=0,1,2, \ldots N_{y}-1$. Similarly, via the inverse Fourier transform, $f(x, y)$ is given by the expression

$$
\begin{aligned}
f(x, y) & =\mathfrak{s}^{-1}\{F(u, v)\} \\
& =\frac{1}{N_{x} N_{y}} \sum_{u=0}^{N_{x}-1} \mathrm{e}^{j 2 \pi u x / N_{x}} \sum_{v=0}^{N_{y}-1} F(u, v) \mathrm{e}^{j 2 \pi v y / N_{y}}
\end{aligned}
$$

for $x=0,1,2, \ldots N_{x}-1$ and $y=0,1,2, \ldots N_{y}-1$. This DFT analysis can extract the individual energy values for the periodic patterns and the defect pattern in the frequency domain. A proper mask filter can discriminate these energy values, and using an inverse DFT, the original defect patterns can be reconstructed.

If the periodic pattern has periods $T_{x}$ and $T_{y}$ along the $x$ and $y$ axes, respectively, their fundamental frequency periods are $\omega_{x}=\Delta u=2 \pi / T_{x}$ and $\omega_{y}=\Delta v=2 \pi / T_{y}$, respectively. The masks for the designed multi-band-pass filter are located at the origin, fundamental frequency coordinates, and the coordinates of integer multiples of the fundamental frequency. The golden template of a possibly defective periodic pattern can be generated from the multi-band-pass filter given in (14).

$$
\begin{aligned}
& H(u, v) \\
& \quad= \begin{cases}1 & \forall\left|u-n \frac{2 \pi}{T_{x}}\right| \leq b_{x} \text { and }\left|v-m \frac{2 \pi}{T_{y}}\right| \leq b_{y} \quad n, m=0,1,2 \ldots \\
0 & \text { otherwise }\end{cases}
\end{aligned}
$$

Here, the bandwidth values $b_{x}$ and $b_{y}$ are selected to accommodate the leakage effect of the periodic pattern. That is, the closer the values $N_{x}$ and $N_{y}$ are to the integer multiples of $T_{x}$ and $T_{y}$, respectively, the smaller should be the values of $b_{x}$ and $b_{y}$. In the extreme case, $b_{x} \approx 0$ and $b_{y} \approx 0$ when $T_{x}$ and $T_{y}$ are integer multiples of $N_{x}$ and $N_{y}$, respectively.

In theory, the multi-band-pass filter $H(u, v)$ can be implemented either using the DFT or its convolution mask. Once the golden template is generated using (14), the image with the defects can easily be obtained by comparing the test image against the golden template. Unlike low-pass filters, the present approach can sharply discriminate the energy distributions of the defect pattern and the periodic pattern in the high frequency range so that both images will be sharp and accurate. In addition, the method can still implement defect detection with 1D periodic patterns in the absence of misorientation. Thus, the multi-band-pass filter can be used for both $1 \mathrm{D}$ and $2 \mathrm{D}$ periodic patterns.

\subsection{Reduction of leakage effect by pattern padding}

The smaller the leakage effect, the better the present approach performs. This subsection proposes a technique called 'pattern padding' that can suppress the leakage effect even when $N_{x}$ and $N_{y}$ values differ greatly from integer multiples of $T_{x}$ and $T_{y}$.

It has been established in Sect. 2.2 that the leakage effect reaches its maximum when $N_{x} / T_{x}=\left(2 n_{x}+1\right) / 2$ and $N_{y} / T_{y}=\left(2 n_{y}+1\right) / 2$ for given integer values of $n_{x}$ and $n_{y}$, and vanishes whenever $N_{x} / T_{x}$ and $N_{y} / T_{y}$ are integer values. Hence, the technique of 'pattern padding' is used to enlarge the size of the test image to an $\widehat{N}_{x} \times \widehat{N}_{y}$ image such that both $\widehat{N}_{x} / T_{x}$ and $\widehat{N}_{y} / T_{y}$ are as close to integer values as possible. This can be done by copying a part of the original test image, whether it contains defects or not, and pasting it surrounding the original image so that a new periodic pattern of the size $\widehat{N}_{x} \times \widehat{N}_{y}$ is formed. To implement this process in the two-dimensional image, the last incomplete patterns in the most right and bottom position of an image must be compensated with a complete periodic pattern. The sum square 


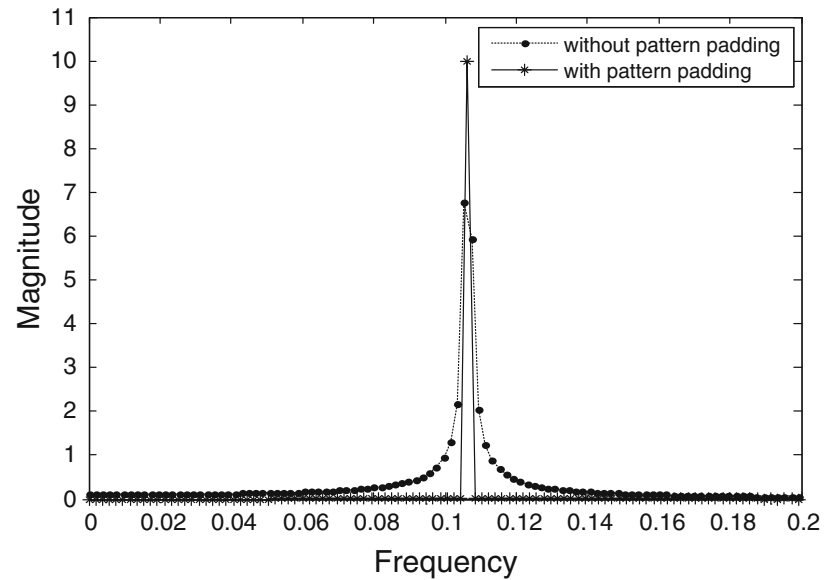

Fig. 4 Comparison of leakage effect with and without pattern padding for data along one axis

difference (SSD) algorithm

$$
\sum_{x=1}^{T_{x}} \sum_{y=1}^{T_{y}}(f(x, y)-p(\bar{x}, \bar{y}))^{2}
$$

is adopted to verify the position, where $f(x, y)$ is an inspection image and $p(\bar{x}, \bar{y})$ is one complete periodic pattern image. The image is extensible in directions $x$ and $y$ until the image size can be near an integer multiple of the pattern.

The effectiveness of pattern padding is shown in the following examples. Figure 4 compares the leakage effect before and after pattern padding. As shown by the solid line, the leakage effect is suppressed to a negligibly small value when the length of the data along one axis is increased from $N_{x} / T_{x}=54.47$ to $\widehat{N}_{x} / T_{x}=55$.

\subsection{Defect detection of periodic patterns using a spatial filter}

The multi-band-pass filter $H(u, v)$ can give very sharp images of defects on a periodic pattern; however, it is computationally demanding. Hence, it should be approximated by a 2D convolution mask of a small size in actual applications in order to save computational effort while obtaining sufficient sharpness in the shapes of the defects.

The $2 \mathrm{D}$ discrete comb function is given by the equation

$h(x, y)=\sum_{m=-\infty}^{\infty} \sum_{n=-\infty}^{\infty} \delta\left(x-n T_{x}\right) \delta\left(y-m T_{y}\right)$.

Consequently, the periodic signal $f(x, y)$ can be expressed as the unit pattern $p(x, y)$ convoluted with the comb function $h(x, y)$ as the following: $f(x, y)=p(x, y) \otimes h(x, y)$.

Here, $\otimes$ denotes the convolution operator. It can also be shown using the discrete convolution theorem [36] that

$F(u, v)=P(u, v) \cdot H(u, v)$.

Here, $H(u, v)$ is the Fourier transform of $h(x, y)$ given by

$H(u, v)=\sum_{m=-\infty}^{\infty} \sum_{n=-\infty}^{\infty} \delta\left(u-n \omega_{x}\right) \delta\left(v-m \omega_{y}\right)$,

where $\omega_{x}=2 \pi / T_{x}$ and $\omega_{y}=2 \pi / T_{y}$. That is, $F(u, v)$ is the product of $P(u, v)$ and the comb function $H(u, v)$ in the frequency domain. In other words, $H(u, v)$ has an intensity value of 0 everywhere except at the origin $(u=0, v=0)$ and the coordinates of the fundamental frequency and its integer multiples in the 2D frequency domain. The corresponding amplitudes of the frequencies are decided by the shape and size of the unit pattern $P(u, v)$.

Accordingly, a defect-free $2 \mathrm{D}$ periodic signal can be obtained from an input image using a comb filter $h(x, y)$. Subsequently, the defects can easily be obtained by comparing the defect free periodic signal with the test image. Moreover, in this approach, the complete spectrum of the defect free periodic signal is retained; hence, the signal corresponding to the defects can be extremely sharp with almost no blurring effects at the edges of the defects.

In actual applications, the ideal multi-band-pass filter $H(u, v)$ is first decomposed into an ideal comb filter $H_{1}(u, v)$, an ideal low-pass filter $H_{\overline{2}}(u, v)$, and the dc-gain $F(0,0)$ of an image $f(x, y)$, as depicted in Fig. 5. Subsequently, the multi-band-pass filter can be realized in the spatial domain. This is expressed as the following equation

$H(u, v)=H_{1}(u, v)-H_{\overline{2}}(u, v)+F(0,0)$.

The advantage with this implementation scheme lies in the fact that the comb filter $H_{1}(u, v)$ can easily be obtained by the up-sampling of a moving average filter [36]. The moving average filter $f_{\mathrm{MA}}$ is a finite impulse response (FIR) filter involving only one parameter, i.e., the filter length $L$, as given by the equation

$f_{\mathrm{MA}}=[1111 \ldots 11] / L$.

The comb filter $f_{\text {Comb }}$ can be designed by the up-sampling of the moving average filter, as given by the equation

$f_{\mathrm{Comb}}=(\uparrow u) f_{\mathrm{MA}}$

The method inserts a value of 0 between the coefficients of the moving average filter. For instance, if the moving average filter is given by $f_{3}=[111] / 3$, then the comb filters of the second, third, fourth orders of up-sampling are related to the moving average filter by the relations $(\uparrow 2) f_{3}=\left[\begin{array}{lllll}1 & 0 & 1 & 0 & 1\end{array}\right] / 3,(\uparrow \quad 3) f_{3}=\left[\begin{array}{lllllll}1 & 0 & 0 & 1 & 0 & 0 & 1\end{array}\right] / 3$ and $(\uparrow 4) f_{3}=\left[\begin{array}{lllllllll}1 & 0 & 0 & 0 & 1 & 0 & 0 & 0 & 1\end{array}\right] / 3$, respectively. 


\section{ㄴ.

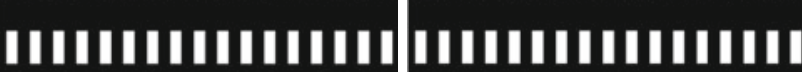

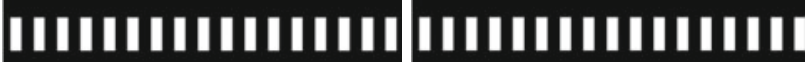

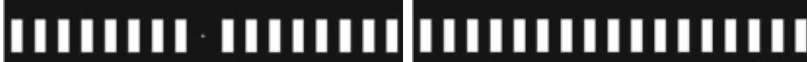

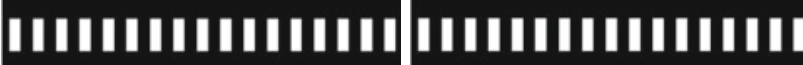

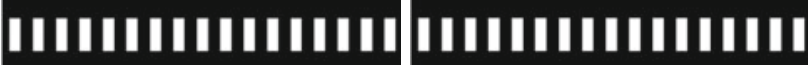

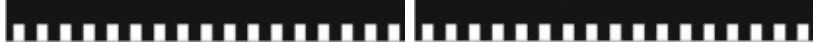

(a)

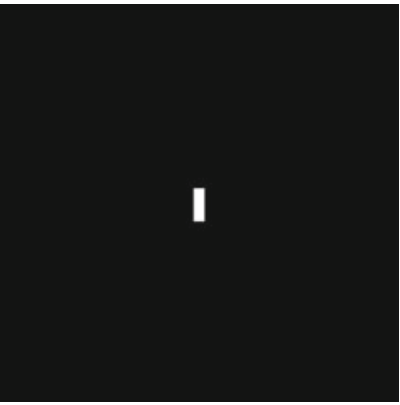

(c) (b)

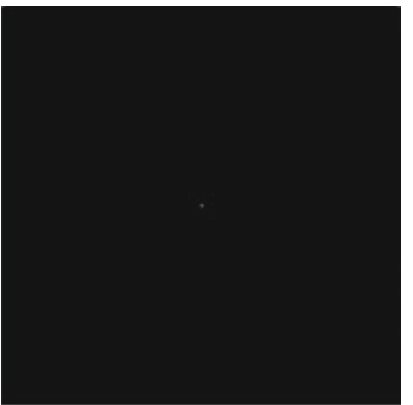

(d)
Fig. 5 Decomposition of the multi-band-pass filter. a Expected filter. b Ideal comb filter $H_{1}(u, v)$. c Ideal low-pass filter $H_{\overline{2}}(u, v)$. d Spectrum center $F(0,0)$

For example, in order to realize the multi-band-pass filter, the bandwidths of the $2 \mathrm{D}$ moving average filter are chosen as $0.5 \omega_{x}$ and $0.5 \omega_{y}$, and a $3 \times 3$ matrix

$f_{M A}=\left[\begin{array}{lll}1 & 1 & 1 \\ 1 & 1 & 1 \\ 1 & 1 & 1\end{array}\right] / 9$

is used to approximate the moving average filter. Furthermore, let the comb filter $H_{1}(u, v)$ and its associated convolution mask $1 h_{1}(x, y)$ be the up-sampled moving average filter by the orders of $u_{x}=17$ and $u_{y}=6$ as given below.

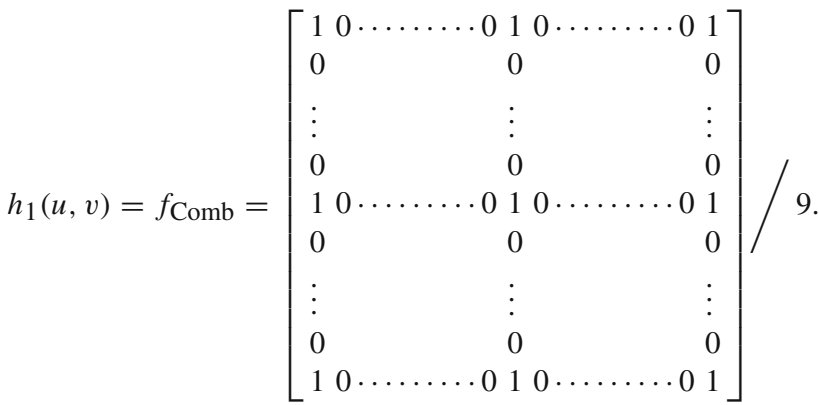

The result of this approximation in the frequency domain is shown in Fig. 6a. With respect to the low-pass filter $H_{2}(u, v)$, we need a moving average filter of sufficient length with a very narrow bandwidth. This can result in large amounts of computational effort. In order to reduce computational effort, we can sequentially pass the signal through convolution

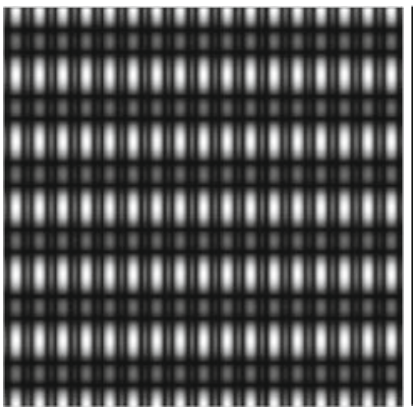

(a)

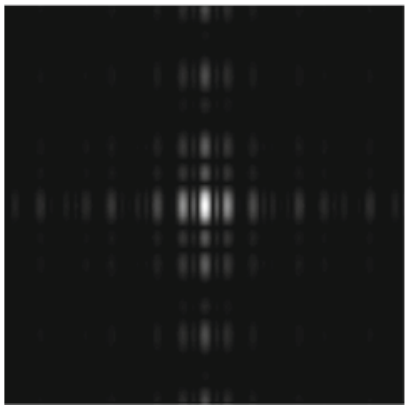

(b)
Fig. 6 Frequency response of $H_{1}(u, v)$ and $H_{\overline{2}}(u, v)$. a Comb filter $H_{1}(u, v)$. b Low-pass filter $H_{2}(u, v)$

masks $h_{1}(x, y)$ and $h_{2}(x, y)$. The two convolution masks are combined to form a sufficiently narrow low-pass filter $H_{2}(u, v)$. In the above examples, the convolution mask 2 $h_{2}(x, y)$ can be reduced to a $5 \times 11$ moving average filter, and via these two convolution masks, the low-pass filter $H_{2}(u, v)$ can be realized. This is shown in Fig. 6b. Therefore, the overall approach needs only two convolution masks to realize an equivalent multi-band-pass filter in the spatial domain.

\subsection{Numerical efficiency of the present approach}

The applied multi-band-pass filter requires large amounts of computational effort in the frequency domain. For example, the fast Fourier transforms (FFT) require four complex number multipliers and eight complex number additions for one pixel in a $256 \times 256$ pixel image (seen in Table 1 ); in addition, its inverse transformation also requires an identical number of computations. This means that a total of 8 complex number multipliers and 16 complex number additions are required for one pixel in a $256 \times 256$ pixel image. Furthermore, one complex number multiplier requires four real number multipliers and two real number additions and one complex number addition requires two real number additions. This means that the frequency filter computation requires 32 real number multipliers and 48 real number additions for one pixel. However, for the same image size, the direct spatial filter uses only the two convolution masks $h_{1}(x, y)$ and $h_{2}(x, y)$. The computations required in this case are $1+1$ real number multipliers and $9+55$ real number additions for one pixel. The computations in the frequency domain are 32 multipliers

Table 1 Calculation order of 2D fast Fourier transform (FFT)

\begin{tabular}{lll}
\hline Image size & $\begin{array}{l}\text { Complex multiplier } \\
\text { order }\end{array}$ & $\begin{array}{l}\text { Complex addition } \\
\text { order }\end{array}$ \\
\hline$N \times N$ & $\left(N^{2} / 2\right) \log _{2} N$ & $N^{2} \log _{2} N$ \\
\hline
\end{tabular}


and 48 additions in total; for the spatial domain, the corresponding numbers are 2 and 64. The spatial filter requires $7 \%(\fallingdotseq 2 / 32)$ less computational effort than that of the frequency filter with regard to real number multiplier calculations. The method not only provides an inspection performance identical to other methods, but it also saves a large amount of computational time. Besides, the proposed spatial filter is also superior to the die-to-die method [8] that requires $9+9$ real number multipliers for linear interpolation in defining the building blocks and building a defect-free image. The method can thus immensely benefit applications for AOI and AVI.

\section{Experimental results}

In Sect. 4.1, we report defect detection in periodic patterns using an multi-band-pass filter in the frequency domain. Further, we reconstruct the image of a defect-free periodic pattern and extract a sharp defect image. The illustrative examples show that the multi-band-pass filter using pattern padding exhibits a performance superior to that of the lowpass filter in the frequency domain.

In Sect. 4.2, it is seen that the presented spatial convolution mask can approximate the aforementioned multi-bandpass filter. We show that this convolution mask can extract sharp defect images with significantly decreased computational effort. Thus, we prove that the method is suitable for actual industrial applications.

Finally, Sect. 4.3 demonstrates the robustness of defect detection in periodic patterns at different luminosities and misorientation angles of the device under inspection. This makes the method suitable for varied industrial applications.

\subsection{Defect detection in periodic patterns using multi-band-pass filter in frequency domain}

The application of the multi-band-pass filter method in the frequency domain involves a number of processes. These processes include the application of FFTs, period evaluation, filter mask design, pattern padding, inverse FFT process, and setting of a binary threshold. In order to simultaneously inspect micro and macro defects, as observed in Fig. 7a-d, we chose the bandwidth values of the selected filter mask to be $b_{x}=5 \cdot(2 \pi / 256)(\mathrm{rad} / \mathrm{pixel})$ and $b_{y}=$ $5 \cdot(2 \pi / 256)(\mathrm{rad} / \mathrm{pixel})$. Subsequently, we applied this multiband-pass filter to the test image with defects in the frequency domain and via inverse FFT, obtained a defect-free image with pure periodic patterns. This image when subtracted with the original test image resulted in a defect image with a background having a grey level value between $-42 \sim 85$. In order to enhance image contrast, the process of a statistical binary threshold was applied to this differential image. The mean $(m)$ and standard deviation $(\sigma)$ of the intensity about the differential image was obtained by histogram analysis. Based on this statistical analysis, the optimal value of the binary threshold was selected as $m+4 \sigma$. Figure 7 shows the resulting grey and binary images with acceptable defects, hole defects, dust defects, and scratch defects.

Figure 8 shows a comparison between operation of a multi-band-pass filter and a low-pass filter implemented in the frequency domain. To describe the performance clearly, the accurate size of the detected defect areas according to various approaches can be adopted to describe the blurring effects as shown in Table 2. When the blurring effects appear, the defects have more area size. Besides, the numbers of connected defect components among the varied approaches can be adopted to describe the appearance of defects as shown in Table 3. The exact numbers of connected defect components have fewer defects with false alarm and escape statistically. As observed in Table 2, the areas of defects extracted by the low-pass filter, corresponding to Fig. 8j-1, are larger than those extracted by the multi-band-pass filter, corresponding to Fig. $8 \mathrm{f}-\mathrm{h}$. As observed in Table 3, the numbers of connected defect components differ greatly from the actual value in the case of the low-pass filter. The multi-band-pass filter in the frequency domain is thus superior to the low-pass filter in terms of extraction of micro defects. Furthermore, Fig. 9 shows that the use of pattern padding can decrease the leakage effects in the image. In Fig. 9e-h, the position located by circle marks indicate noise areas (not defects) in the circumference of the inspection images without pattern padding. In Fig. 9i-1, the circles marks indicate more micro defects and the previous noise areas disappear with pattern padding. Therefore, the process of pattern padding can result in the more accurate detection and location of micro defects.

\subsection{Defect detection of periodic patterns using multi-band-pass filter in spatial domain}

In addition, we verify the application of the multi-bandpass filter method in the spatial domain. This process includes period evaluation, convolution mask implementation, pattern padding, and setting of a binary threshold. The main difference in the processes involved in the frequency and spatial domains is the use of the convolution mask, whose design is described in Sect. 3.3. Even in the spatial domain, we show that the use of the multi-band-filter allows for a more accurate detection of micro and macro defects and gives better inspection results (without blurring effects) than those obtained using the low-pass filter. Tables 2 and 3 show that the areas and numbers of connected 


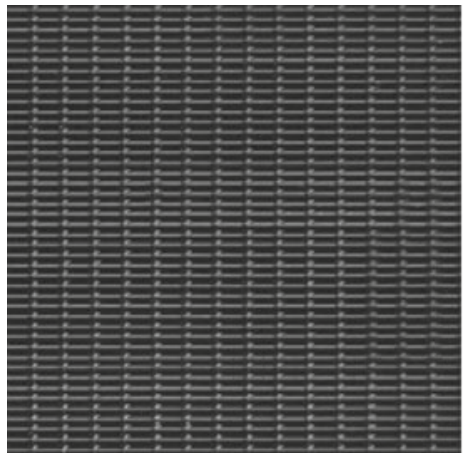

(a)

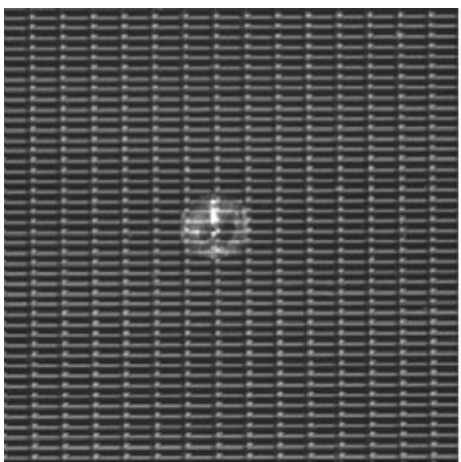

(b)

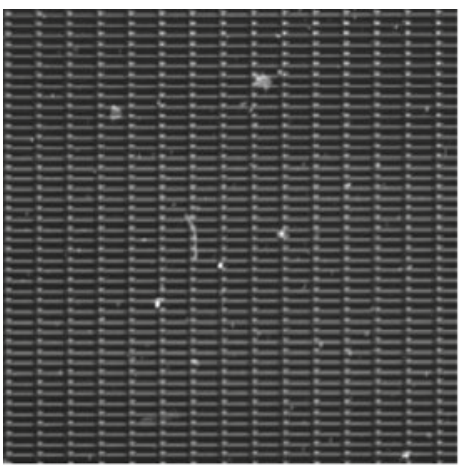

(c)

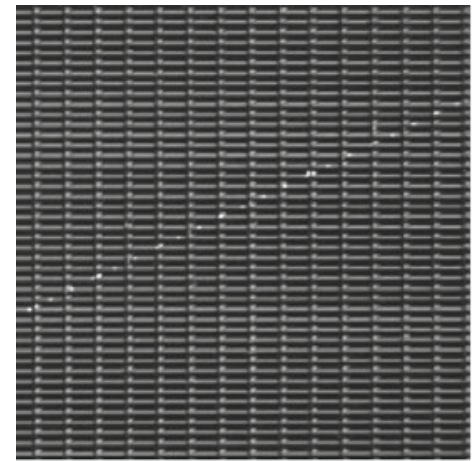

(d)

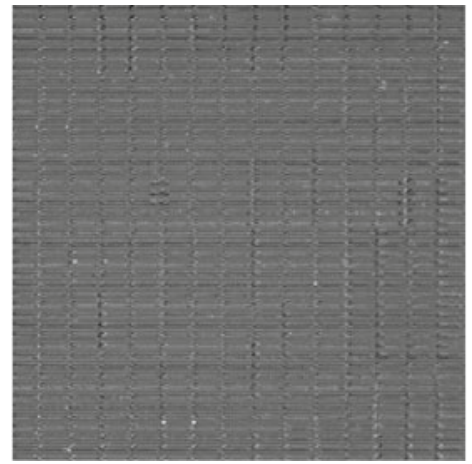

(e)

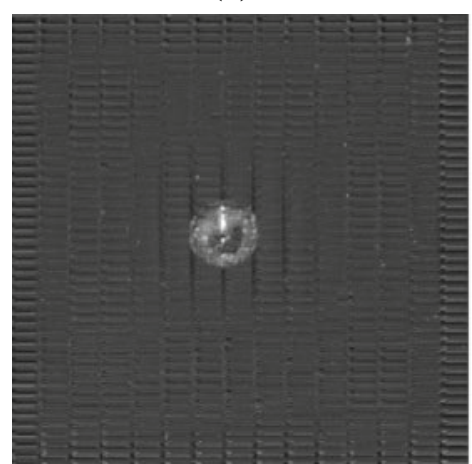

(f)

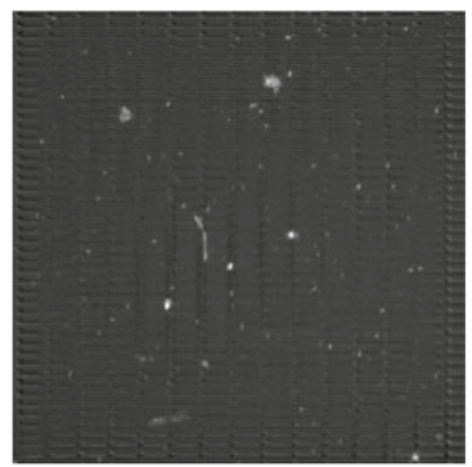

(g)

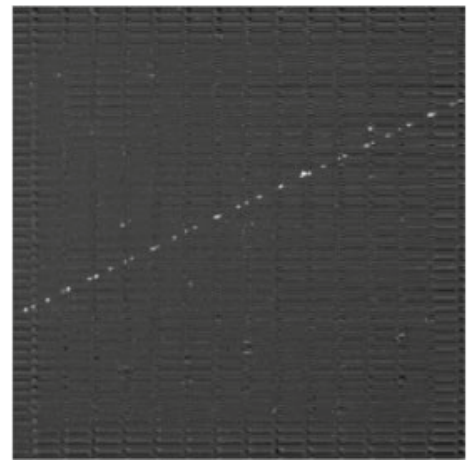

(h)

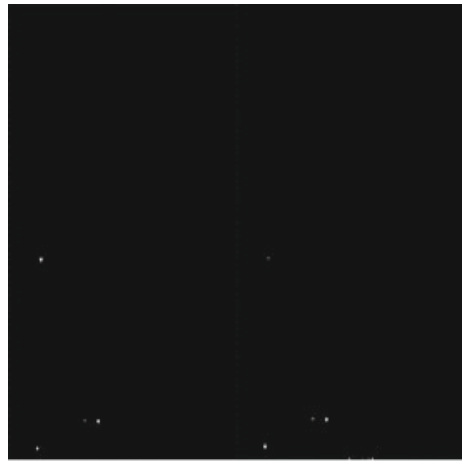

(i)

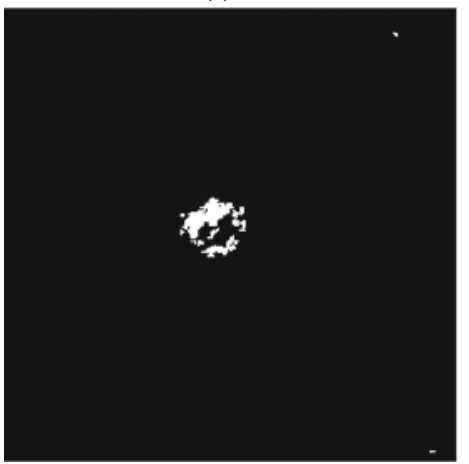

(j)

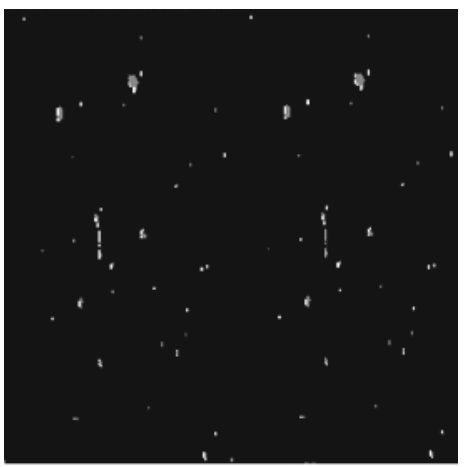

(k)

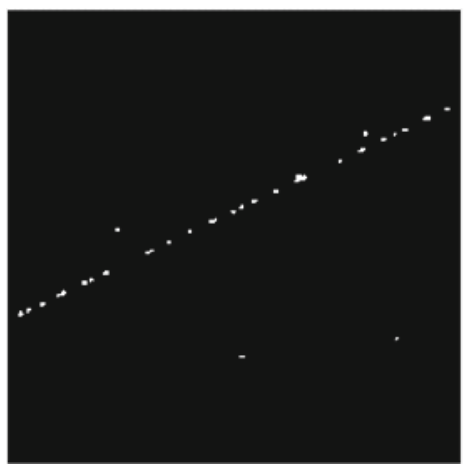

(l)
Fig. 7 Results of defect inspection using reported multi-band-pass filter. a Image 1 with acceptable defect. b Image 2 with hole defect. c Image 3 with dust defect. d Image 4 with scratch defect. e Grey image of defects. f Grey image of defects. g Grey image of defects. $\mathbf{h}$ Grey image of defects. i Binary image of defects. j Binary image of defects. $\mathbf{k}$ Binary image of defects. I Binary image of defects 


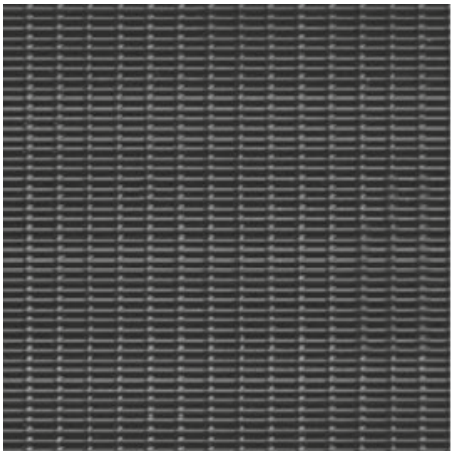

(a)

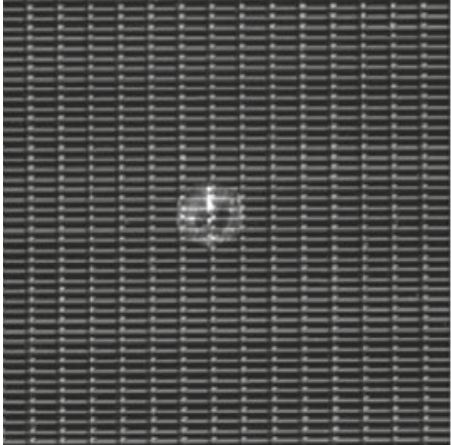

(b)

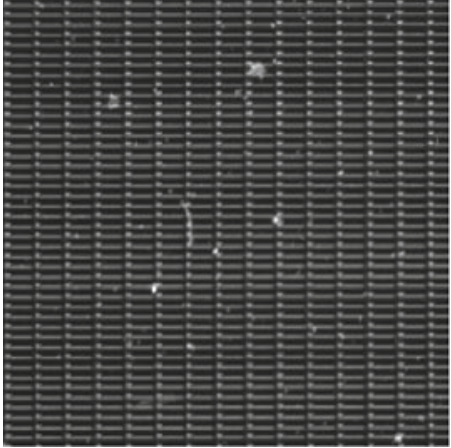

(c)

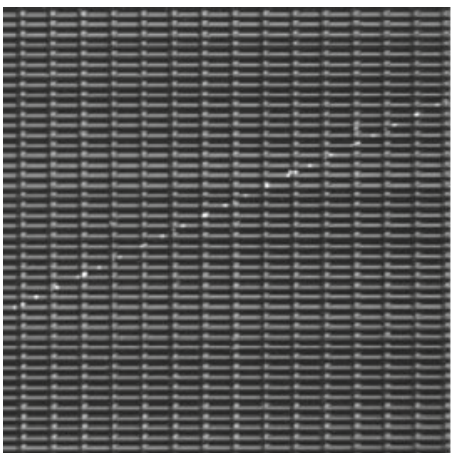

(d)

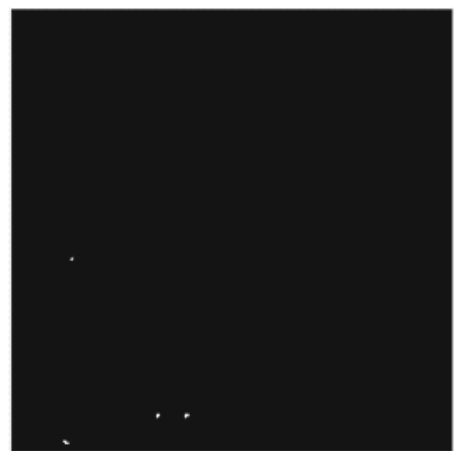

(e)

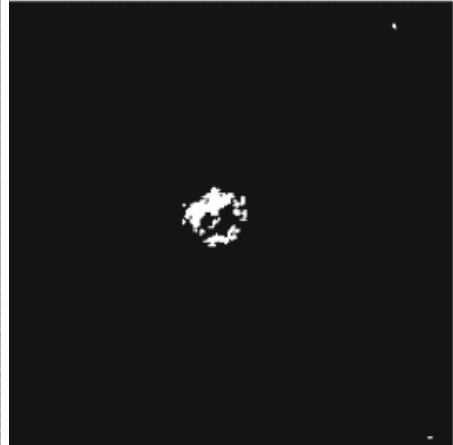

(f)

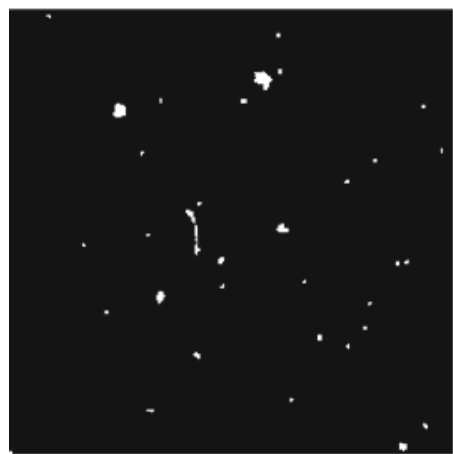

(g)

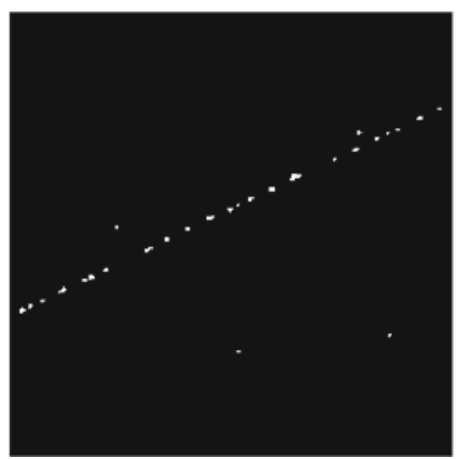

(h)

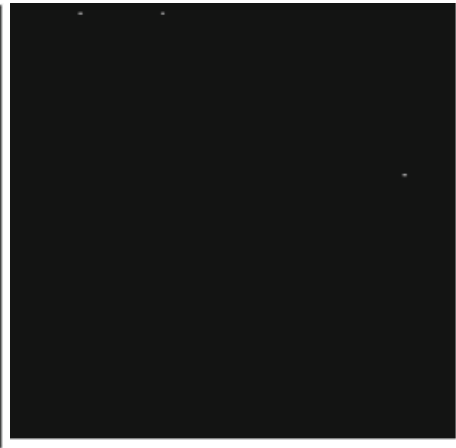

(i)

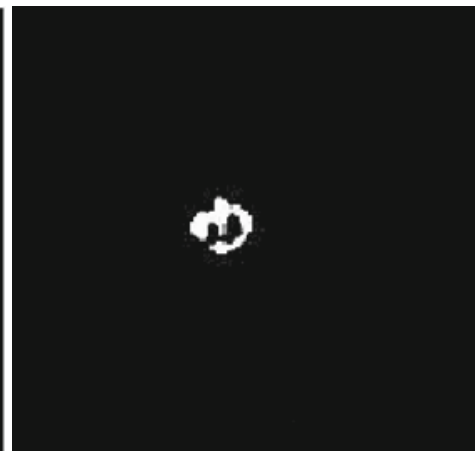

(j)

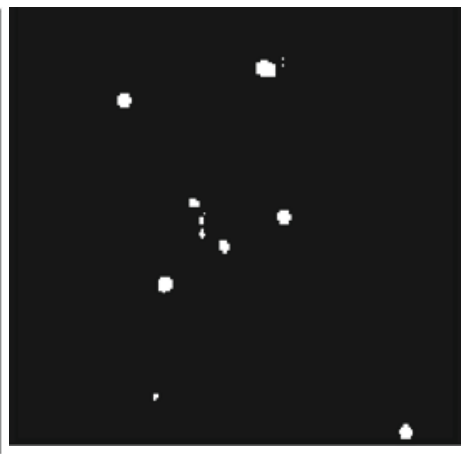

(k)

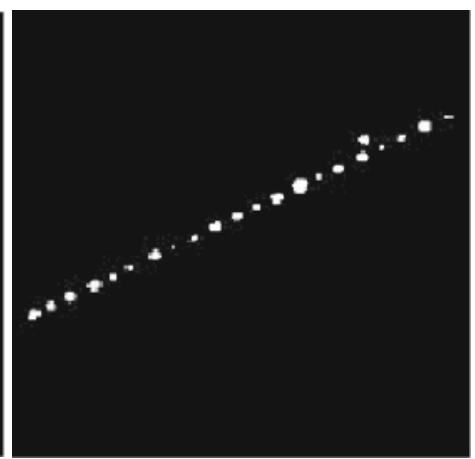

(l)
Fig. 8 Comparison between operation of multi-band-pass filter implemented in frequency domain and low-pass filter. a Image 1 with acceptable defect. b Image 2 with hole defect. c Image 3 with dust defect. d Image 4 with scratch defect. e Multi-band-pass filter in frequency domain. f Multi-band-pass filter in frequency domain. g Multi-bandpass filter in frequency domain. $\mathbf{h}$ Multi-band-pass filter in frequency domain. i Low-pass filter. $\mathbf{j}$ Low-pass filter. $\mathbf{k}$ Low-pass filter. I Low-pass filter 
Table 2 Comparison of detected defect areas among multi-band-pass filter in frequency domain, multi-band-pass filter in spatial domain and low-pass filter
Table 3 Numbers of connected defect components detected using multi-band-pass filter in frequency domain, multi-band-pass filter in spatial domain and low-pass filter

\begin{tabular}{lllll}
\hline Performance & \multicolumn{4}{l}{ Size of detected defect areas (pixels) } \\
\cline { 2 - 5 } Methods & Image 1 & Image 2 & Image 3 & Image 4 \\
\hline Actual defect judged by human eyes & 12 & 325 & 265 & 110 \\
Multi-band-pass filter in frequency domain & 12 & 320 & 261 & 107 \\
Multi-band-pass filter in spatial domain & 10 & 318 & 259 & 101 \\
Low-pass filter & 1 & 351 & 294 & 150 \\
\hline
\end{tabular}

\begin{tabular}{lllll}
\hline Performance & \multicolumn{4}{l}{ Numbers of connected defect components } \\
\cline { 2 - 5 } Methods & Image 1 & Image 2 & Image 3 & Image 4 \\
\hline Actual defect judged by human eyes & 4 & 13 & 36 & 29 \\
Multi-band-pass filter in frequency domain & 4 & 13 & 34 & 28 \\
Multi-band-pass filter in spatial domain & 2 & 22 & 35 & 34 \\
Low-pass filter & 1 & 1 & 13 & 22 \\
\hline
\end{tabular}

defect components obtained using the multi-band-pass filter, corresponding to Fig. $10 \mathrm{e}-\mathrm{h}$, is more accurate than that obtained for the low-pass filter, corresponding to Fig. 10i-1. It is noteworthy that the multi-band-pass filter implemented in the spatial domain, corresponding to Fig. 10e-h, provides poor results when compared with its operation in the frequency domain, corresponding to Fig. 8e-h. This is because larger masks used in the comb filter (in the spatial domain) lead to the preservation of the relatively less defect energy existing at lower frequencies when compared with the defect energies encountered in the frequency domain operation. The actual defects will be broken into a number of smaller defects, which will result in a larger number of connected defect components with smaller area. Therefore, it is important to adequately select convolution mask implemented in the spatial domain; this factor can otherwise offset the significant decrease in calculation time.

\subsection{Performance of defect detection in periodic patterns with different luminosities and misorientations}

In industrial applications, image luminosity problems and misorientations of the inspected device usually occur in the defect detection process. The image luminosity and the device misorientations depend on the environmental lighting and mechanical alignment, respectively. In our approach, this problem is addressed as described below.

We individually processed 150 periodic pattern samples with different luminosities and small tilt angles (misorientations) through two multi-band-pass filter processes in frequency and spatial domains. Owing to our use of the multi-band-pass filter, we were able to thoroughly remove the pattern energies existing at higher frequencies along with the dc energy. The defect energy existing at lower frequencies was preserved completely. Further, changes in luminosity values did not affect our proposed method in the frequency and spatial domains. This was proved by the fact that 150 test images with 3 different defects captured at random luminosity values showed identical defect areas and numbers of connected defect components.

When the tilt angles vary within a small range of $0^{\circ}-2^{\circ}$, the multi-band-pass filter is rotated with the corresponding angles in the frequency and spatial domains. Table 4 shows that defects can still be thoroughly extracted within area error values of three pixels in the frequency domain method. In addition, Table 5 shows that the number of connected defect components remains exactly the same at five different angles. Table 6 shows that the defects can still be thoroughly extracted within area error values of five pixels in the spatial domain method. Further, Table 7 shows that the number of connected defect components at five different angles remains exactly the same. Therefore, the multi-bandpass filter can provide a superior and robust defect detection technique in terms of different image luminosity values and misorientations of the device under inspection.

\section{Conclusions}

In this study, we proposed and implemented a multiband-pass filter using pattern padding for inspecting 


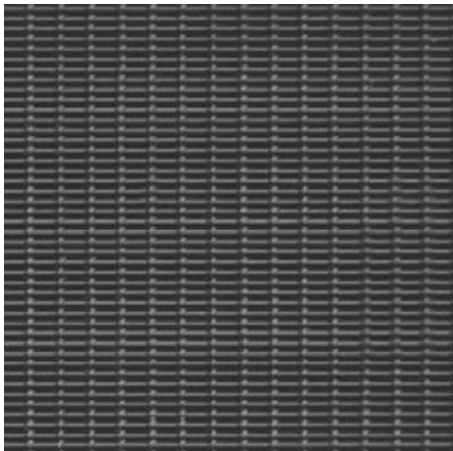

(a)

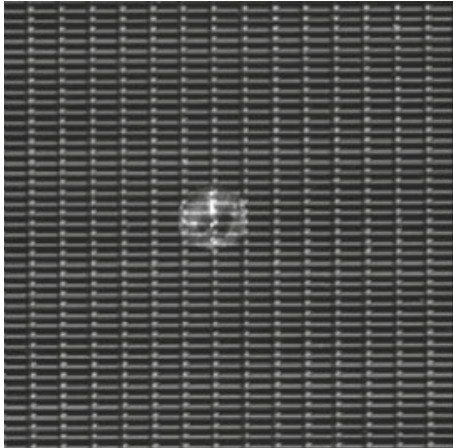

(b)

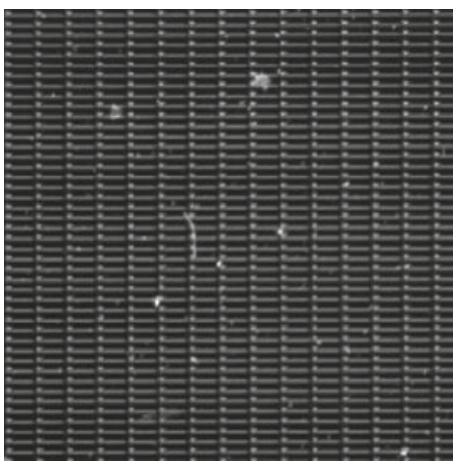

(c)

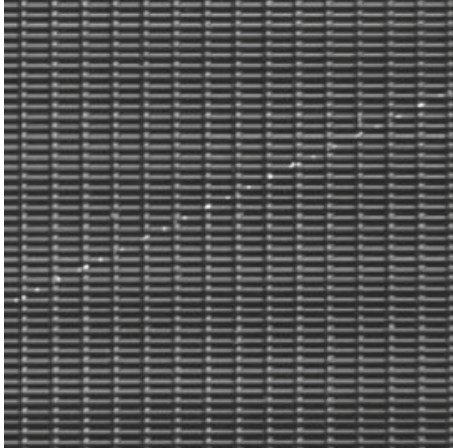

(d)

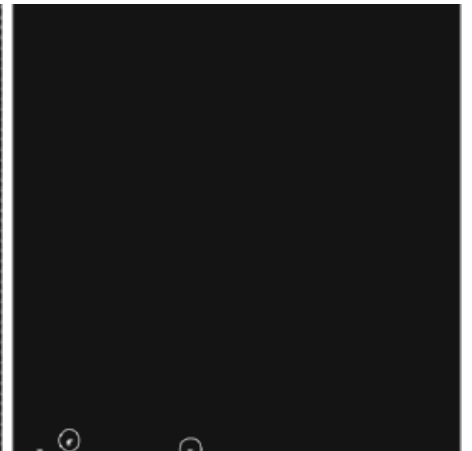

(e)

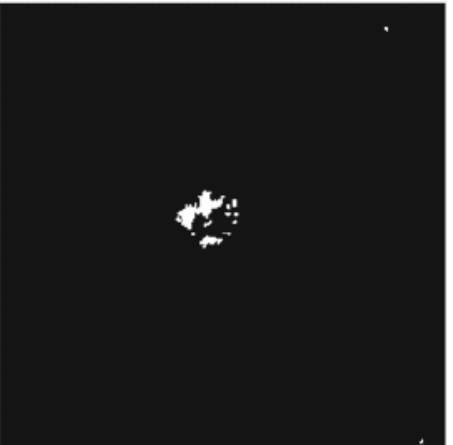

(f)

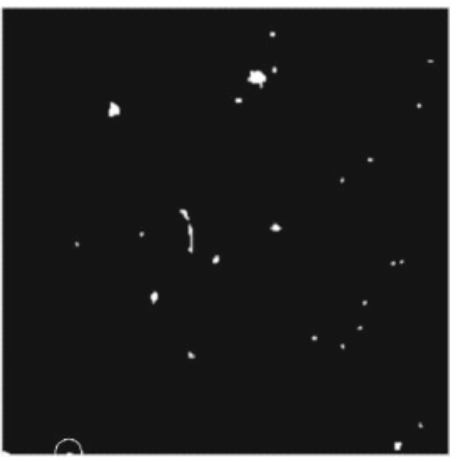

(g)

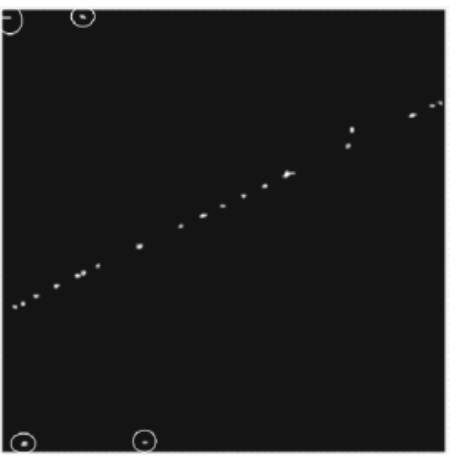

(h)

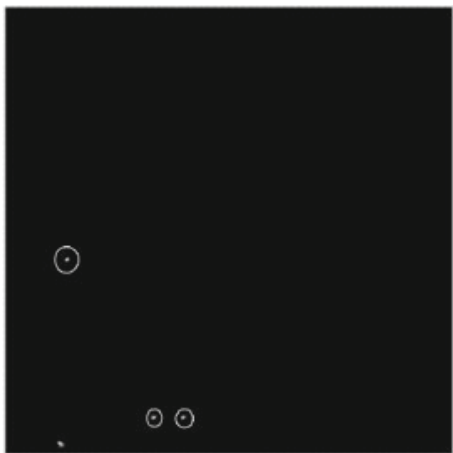

(i)

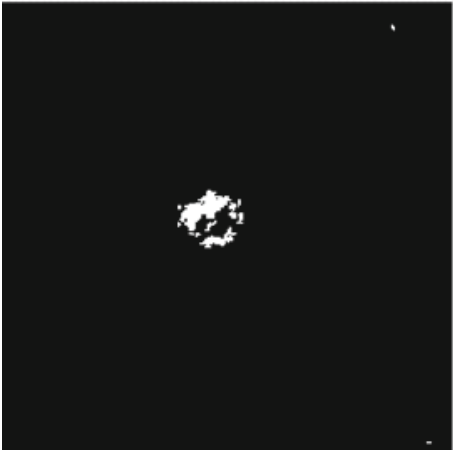

(j)

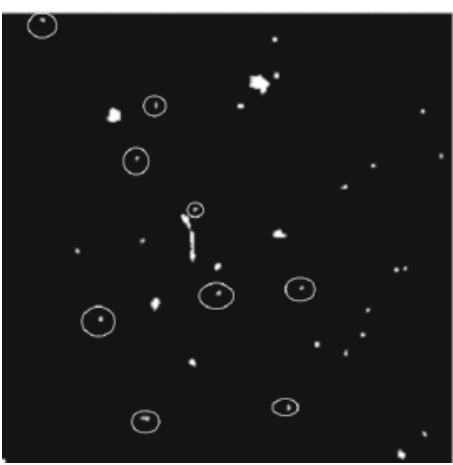

(k)

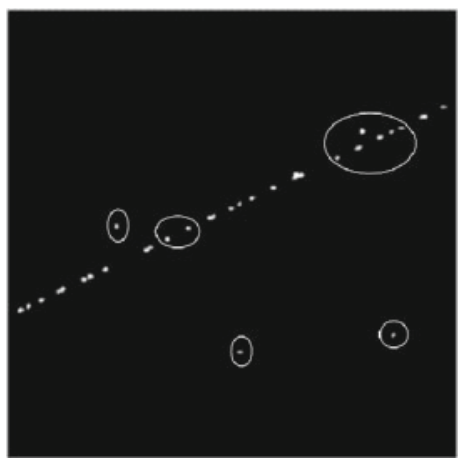

(l)
Fig. 9 Comparison of multi-band-pass filter operation with and without pattern padding. a Image 1 with acceptable defect. b Image 2 with hole defect. c Image 3 with dust defect. d Image 4 with scratch defect. e Without pattern padding. $\mathbf{f}$ Without pattern padding. g Without pattern padding. $\mathbf{h}$ Without pattern padding. $\mathbf{i}$ With pattern padding. $\mathbf{j}$ With pattern padding. $\mathbf{k}$ With pattern padding. I With pattern padding 


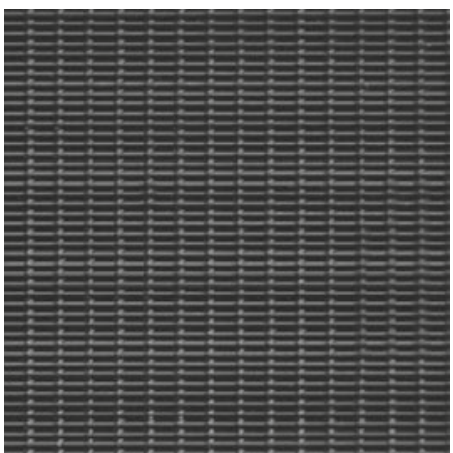

(a)

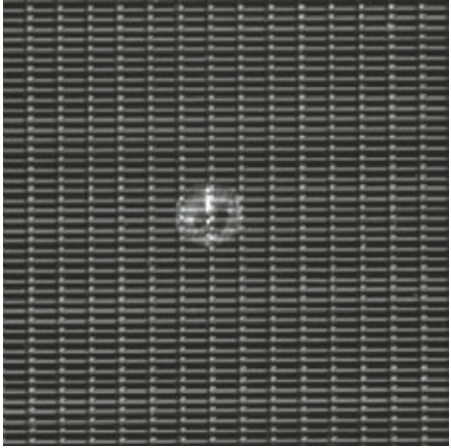

(b)

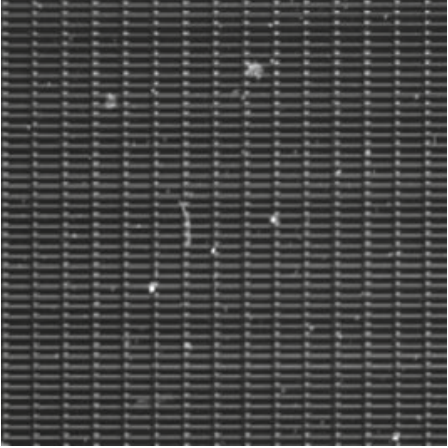

(c)

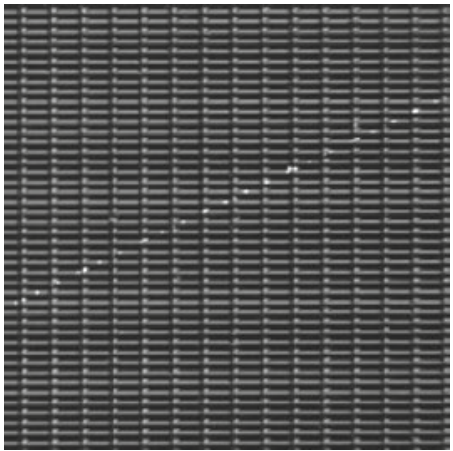

(d)

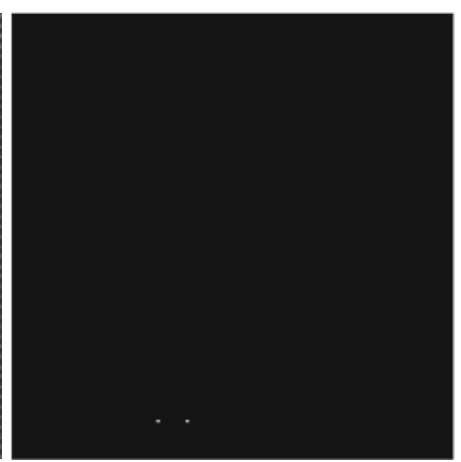

(e)

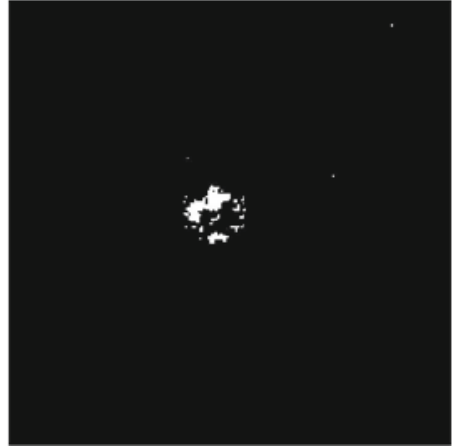

(f)

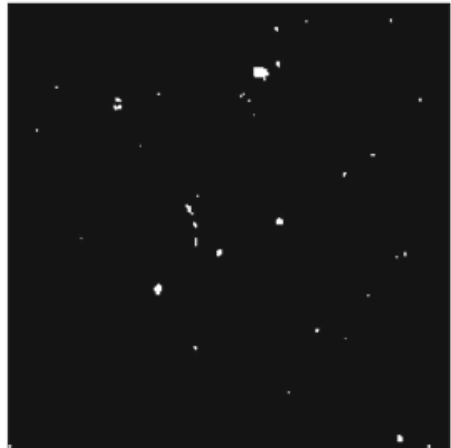

(g)

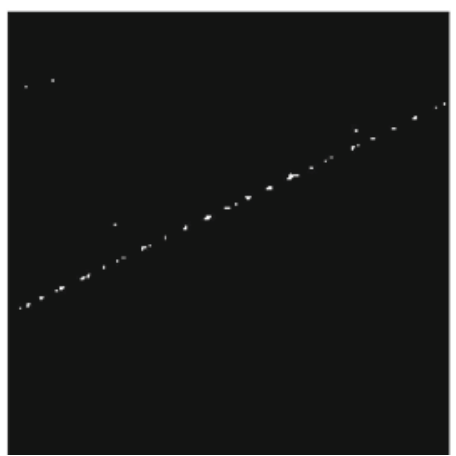

(h)

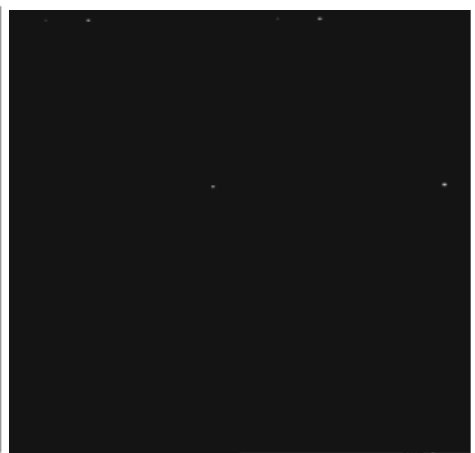

(i)

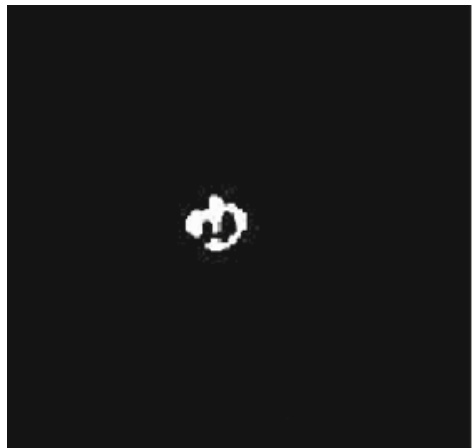

(j)

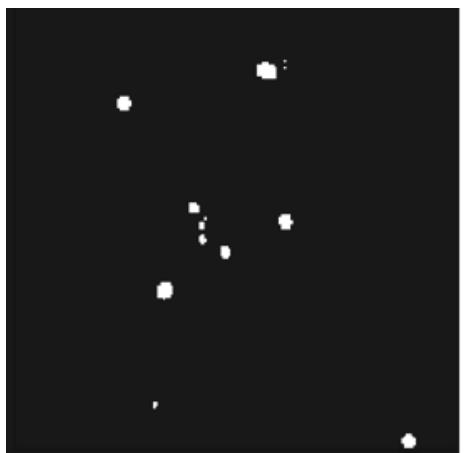

(k)

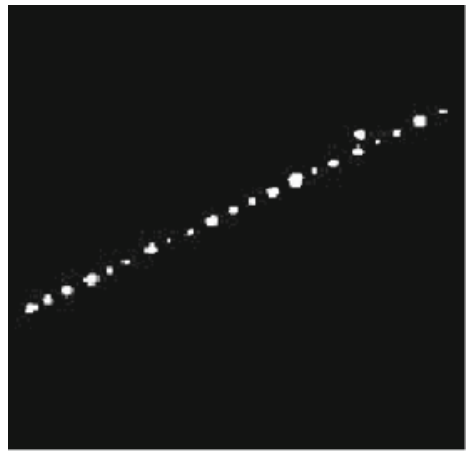

(l)
Fig. 10 Comparison between operation of multi-band-pass filter implemented in spatial domain and low-pass filter. a Image 1 with acceptable defect. b Image 2 with hole defect. c Image 3 with dust defect. d Image 4 with scratch defect. e Multi-band-pass filter in spa- tial domain. f Multi-band-pass filter in spatial domain. g Multi-bandpass filter in spatial domain. $\mathbf{h}$ Multi-band-pass filter in spatial domain. $\mathbf{i}$ Low-pass filter. $\mathbf{j}$ Low-pass filter. $\mathbf{k}$ Low-pass filter. $\mathbf{l}$ Low-pass filter 
Table 4 Detected defect areas among different tilt angles using multiband-pass filter in frequency domain

\begin{tabular}{lllll}
\hline \multirow{2}{*}{$\begin{array}{l}\text { Terformance } \\
\text { Tilt angle }\end{array}$} & \multicolumn{4}{l}{ Size of detected defect areas (pixels) } \\
\cline { 2 - 5 } & Image 1 & Image 2 & Image 3 & Image 4 \\
\hline $0^{\circ}$ & 12 & 320 & 261 & 107 \\
$0.5^{\circ}$ & 12 & 321 & 262 & 105 \\
$1^{\circ}$ & 11 & 320 & 260 & 106 \\
$1.5^{\circ}$ & 11 & 322 & 259 & 105 \\
$2^{\circ}$ & 11 & 321 & 259 & 104 \\
\hline
\end{tabular}

Table 5 Numbers of connected defect components detected for different tilt angles using multi-band-pass filter in frequency domain

\begin{tabular}{lllll}
\hline \multirow{2}{*}{$\begin{array}{l}\text { Performance } \\
\text { Tilt angle }\end{array}$} & \multicolumn{4}{l}{ Numbers of connected defect components } \\
\cline { 2 - 5 } & Image 1 & Image 2 & Image 3 & Image 4 \\
\hline $0^{\circ}$ & 4 & 13 & 34 & 28 \\
$0.5^{\circ}$ & 4 & 13 & 34 & 28 \\
$1^{\circ}$ & 4 & 13 & 34 & 28 \\
$1.5^{\circ}$ & 4 & 13 & 34 & 28 \\
$2^{\circ}$ & 4 & 13 & 34 & 28 \\
\hline
\end{tabular}

Table 6 Detected defect areas among different tilt angles using multiband-pass filter in spatial domain

\begin{tabular}{lllll}
\hline Performance & \multicolumn{4}{l}{ Size of detected defect areas (pixels) } \\
\cline { 2 - 5 } Tilt angle & Image 1 & Image 2 & Image 3 & Image 4 \\
\hline $0^{\circ}$ & 10 & 318 & 259 & 101 \\
$0.5^{\circ}$ & 10 & 315 & 257 & 99 \\
$1^{\circ}$ & 10 & 314 & 255 & 99 \\
$1.5 \circ$ & 9 & 315 & 254 & 99 \\
$2^{\circ}$ & 9 & 313 & 254 & 98 \\
\hline
\end{tabular}

Table 7 Numbers of connected defect components detected for different tilt angles using multi-band-pass filter in spatial domain

\begin{tabular}{lllll}
\hline Performance & \multicolumn{4}{l}{ Numbers of connected defect components } \\
\cline { 2 - 5 } Tilt angle & Image 1 & Image 2 & Image 3 & Image 4 \\
\hline $0^{\circ}$ & 2 & 22 & 35 & 34 \\
$0.5^{\circ}$ & 2 & 22 & 35 & 34 \\
$1^{\circ}$ & 2 & 22 & 35 & 34 \\
$1.5^{\circ}$ & 2 & 22 & 35 & 34 \\
$2^{\circ}$ & 2 & 22 & 35 & 34 \\
\hline
\end{tabular}

periodic patterns with micro and macro defects. Our technique exhibits an implicit defect-free reference image that avoids scaling and alignment procedures. Further, the retention of the high frequency spectrum of the defects reduces the blurring effects and produces a sharp defect image. Besides, the method reduces computational effort using spatial convolution masks. In addition, the robustness of this method of inspection is vastly improved in terms of variations in the sample luminosity values and tilt angles of the inspected device. In conclusion, this study provides a rapid and reliable technique for the inspection of defects and irregularities in periodic patterns for various industrial applications.
Acknowledgments This author gratefully acknowledges valuable suggestions from the reviewers.

Open Access This article is distributed under the terms of the Creative Commons Attribution License which permits any use, distribution, and reproduction in any medium, provided the original author(s) and the source are credited.

\section{References}

1. Meisburger, W.D., Brodie, A.D., Desai, A.A.: Low-voltage electronic-optical system for the high-speed inspection of integrated circuits. J. Vac. Sci. Technol. Ser. B 10, 2804-2808 (1992)

2. Shankar, N.G., Zhong, Z.W.: A rule-based computing pproach for the segmentation of semiconductor defects. Microelectron. J. 37, 500-509 (2006)

3. Shankar, N.G., Zhong, Z.W.: Defect detection on semiconductor wafer surfaces. Microelectron. Eng. 77, 337-346 (2005)

4. Khalaj, B.H., Aghajan, H., Paulraj, A., Kailath, T.: Automated direct patterned wafer inspection. Proc. SPIE 1907, 140147 (1993)

5. Khalaj, B.H., Aghajan, H.K., Kailath, T.: Digital image processing techniques for patterned wafer inspection. Proc. SPIE 1926, 508516 (1993)

6. Khalaj, B.H., Aghajan, H., Paulraj, A., Kailath, T.: Defect inspection of periodic patterns with low-order distortions. Proc. SPIE 2183, 13-19 (1994)

7. Khalaj, B.H., Aghajan, H.K., Kailath, T.: Patterned wafer inspection by high resolution spectral estimation techniques. Mach. Vis. Appl. 7, 178-185 (1994)

8. Xie, P., Guan, S.U.: A golden-template self-generating method for patterned wafer inspection. Mach. Vis. Appl. 12, 149-156 (2000)

9. Guan, S.U., Xie, P., Li, H.: A golden-block-based self-refining scheme for repetitive patterned wafer inspections. Mach. Vis. Appl. 13, 314-321 (2003)

10. Tsai, D.M., Chuang, S.T.: 1D-based defect detection in patterned TFT-LCD panels using characteristic fractal dimension and correlations. Mach. Vis. Appl. 20, 423-434 (2009)

11. Tsai, D.M., Lai, S.C.: Defect detection in periodically patterned surfaces using independent component analysis. Pattern Recogn. 41, 2812-2832 (2008)

12. Lu, C.J., Tsai, D.M.: Defect inspection of patterned thin film transistor-liquid crystal display panels using a fast sub-imagebased singular value decomposition. Int. J. Prod. Res. 42, 43314351 (2004)

13. Bourgeat, P., Meriaudeau, F., Tobin, K.W., Gorria, P.: Content based segmentation of patterned wafers. J. Electron. Imaging 13, 428435 (2004)

14. Song, Y.C., Choi, D.H., Park, K.H.: Multiscale detection of defect in thin film transistor liquid crystal display panel. Jpn. J. Appl. Phys. 43, 5465-5468 (2004)

15. Liu, Y.H., Lin, S.H., Hsueh, Y.L., Lee, M.J.: Automatic target defect identification for TFT-LCD array process inspection using kernel FCM-based fuzzy SVDD ensemble. Expert Syst. Appl. 36, 19781998 (2009)

16. Chen, W.C., Hsu, S.W.: A neural-network approach for an automatic LED inspection system. Expert Syst. Appl. 33, 531537 (2007)

17. Lin, H.D.: Automated defect inspection of light-emitting diode chips using neural network and statistical approaches. Expert Syst. Appl. 36, 219-226 (2009)

18. Liu, Y.H., Huang, Y.K., Lee, M.J.: Automatic inline defect detection for a thin film transistor-liquid crystal display array process 
using locally linear embedding and support vector data description. Meas. Sci. Technol. 19, 1-16 (2008)

19. Lin, C.T., Chang, C.L., Cheng, W.C.: A recurrent fuzzy cellular neural network system with automatic structure and template learning. IEEE Trans. Circ. Syst.-I: Regular Papers 51, 10241035 (2004)

20. Chang, C.L., Fan, K.W., Lin, C.T., Cheng, W.C.: A recurrent fuzzy coupled cellular neural network system with automatic structure and template learning. IEEE Trans. Circ. Syst.-I: Express Briefs 53, 1024-1035 (2006)

21. Tsai, D.M., Wu, S.K.: Automated surface inspection using Gabor filters. Int. J. Adv. Manuf. Technol. 16, 474-482 (2000)

22. Kumar, A., Pang, G.K.H.: Defect detection in textured materials using Gabor filters. IEEE Trans. Ind. Appl. 38, 425-440 (2002)

23. Bodnarova, A., Bennamoun, M., Latham, S.: Optimal Gabor filters for textile flaw detection. Pattern Recogn. 35, 2973-2991 (2002)

24. Bourgeat, P., Meriaudeau, F., Tobin, K.W., Gorria, P.: Comparison of texture features for segmentation of patterned wafers. Proc. SPIE 5266, 179-190 (2004)

25. Tsai, D.M., Chiang, C.H.: Automatic band selection for wavelet reconstruction in the application of defect detection. Image Vis. Comput. 21, 413-431 (2003)

26. Ngan, H.Y.T., Pang, G.K.H., Yung, S.P., Ng, M.K.: Wavelet based methods on patterned fabric defect detection. Pattern Recogn. 38, 559-576 (2005)

27. Latif-Ameta, A., Ertuzuna, A., Ercil, A.: Efficient method for texture defect detection: sub-band domain co-occurrence matrices. Image Vis. Comput. 18, 543-553 (2000)

28. Chan, C.H., Pang, G.K.H.: Fabric defect detection by Fourier analysis. IEEE Trans. Ind. Appl. 36, 1267-1276 (2000)

29. Tsai, D.M., Chuang, S.T., Tseng, Y.H.: One-dimensional-based automatic defect inspection of multiple patterned TFT-LCD panels using Fourier image reconstruction. Int. J. Prod. Res. 45, 12971321 (2007)

30. Tsai, D.M., Hung, C.Y.: Automatic defect inspection of patterned thin film transistor-liquid crystal display (TFT-LCD) panels using one-dimensional Fourier reconstruction and wavelet decomposition. Int. J. Prod. Res. 43, 4589-4607 (2005)
31. Tseng, Y.H.: Automatic surface inspection for TFT-LCD array panels using Fourier reconstruction. MS thesis, Dept. Ind. Engg., YuanZe Univ., Taiwan (2002)

32. Wood, E.J.: Applying Fourier and associated transforms to pattern characterization in textiles. Textile Res. J. 60, 212-220 (1990)

33. Ravandi, S.A.H., Toriumi, K.: Fourier transform analysis of plain weave fabric appearance. Textile Res. J. 65, 676-683 (1995)

34. Xie, X.: A review of recent advances in surface defect detection using texture analysis techniques. Electron. Lett. Comput. Vis. Image Anal. 7, 1-22 (2008)

35. Weng, Y.S., Perng, M.H.: Periodic pattern inspection using convolution masks. MVA2007 IAPR Conference on Machine Vision Applications, Tokyo, Japan, pp. 544-547 (May 16-18, 2007)

36. Proakis, J.G., Manolakis, D.G.: Digital Signal Processing. Prentice Hall, India (1996)

37. Oppenheim, A.V., Schafer, R.W.: Discrete-Time Signal Processing, 2nd edn. Prentice Hall, (1999)

\section{Author Biographies}

Zong-Da Tsai received his M.S. degree in mechanical engineering from the National Taiwan University, Taipei, Taiwan, in 1996. He is currently pursuing his Ph.D. degree in machine vision at the Power Mechanical Engineering, National Tsing Hua University and working at the National Synchrotron Radiation Research Center, Hsinchu, Taiwan. He is interested in image processing and industrial inspection.

Ming-Hwei Perng received his Ph.D. degree in control engineering from the Cambridge University, Cambridge, UK, in 1989. From 1990, he headed the research group (precision position and system control lab) at the Power Mechanical Engineering, National Tsing Hua University, Hsinchu, Taiwan and his research interests include machine vision, image processing and control. Currently he is a professor emeritus of Power Mechanical Engineering at the National Tsing Hua University. 